\title{
Some Notes about the Continuous-in-Time Financial Model
}

\author{
Tarik Chakkour \\ Universite Bretagne-Sud, UMR 6205, LMBA, 56000 Vannes, France \\ Correspondence should be addressed to Tarik Chakkour; tarik.chakkour@univ-ubs.fr
}

Received 19 January 2017; Accepted 15 February 2017; Published 21 March 2017

Academic Editor: Sergei V. Pereverzyev

Copyright (C) 2017 Tarik Chakkour. This is an open access article distributed under the Creative Commons Attribution License, which permits unrestricted use, distribution, and reproduction in any medium, provided the original work is properly cited.

In this paper, we investigate the properties of operators in the continuous-in-time model which is designed to be used for the finances of public institutions. These operators are involved in the inverse problem of this model. We discuss this inverse problem in Schwartz space that we prove the uniqueness theorem.

\section{Introduction}

Linear inverse problems arise whenever throughout engineering and the mathematical sciences. In most applications, these problems are ill-conditioned or underdetermined. Consequently, over the last two decades, the theory and practice of inverse problems is rapidly growing, if not exploding in many scientific domains. The fundamental reason is that solutions to inverse problems describe important properties of solution in this theory and the development of sophisticated numerical techniques for its treating on a level of high complexity. We mention the paper [1] introduced by Hadamard in the field of ill-posed problems.

We built in previous work $[2,3]$ the continuous-intime model which is designed to be used for the finances of public institutions. This model uses measures over time interval to describe loan scheme, reimbursement scheme, and interest payment scheme. Algebraic Spending Measure $\widetilde{\sigma}$ and Loan Measure $\widetilde{\kappa}_{E}$ are financial variables involved in the model. Measure $\widetilde{\sigma}$ is defined such that the difference between spending and incomes required to satisfy the current needs. Assume that measures $\widetilde{\sigma}$ and $\widetilde{\kappa}_{E}$ are absolutely continuous with respect to the Lebesgue measure $d t$. This means that they read $\sigma(t) d t$ and $\kappa_{E}(t) d t$, where $t$ is the variable in $\mathbb{R}$. We call $\sigma$ and $\kappa_{E}$ time densities.

Let $F$ and $G$ be normed spaces. Throughout this paper, $\mathscr{L}: F \rightarrow G$ is a continuous linear application (in short, an operator). We say that the following problem:

\section{Given $\sigma \in G$,}

$$
\begin{gathered}
\text { find } \kappa_{E} \in F \\
\text { such that } \sigma=\mathscr{L}\left[\kappa_{E}\right] \text {, }
\end{gathered}
$$

is well-posed if $\mathscr{L}$ is invertible and its inverse $\mathscr{L}^{-1}: G \rightarrow F$ is continuous. In other words, the problem is said to be wellposed if

$$
\sigma=\mathscr{L}\left[\kappa_{E}\right] ; \quad \forall \sigma \in G, \exists ! \kappa_{E} \in F
$$

the solution $\kappa_{E}$ depends continuously on $\sigma$.

Existence and uniqueness of a solution for all $g \in G$ (condition (2)) are equivalent to surjectivity and injectivity of $\mathscr{L}$, respectively. Stability of the solution (condition (3)) amounts to continuity of $\mathscr{L}^{-1}$. Conditions (2) and (3) are referred to as the Hadamard conditions. A problem which is not well-posed is said to be ill-posed. Operator $\mathscr{L}$ links between Algebraic Spending Density $\sigma$ and Loan Density $\kappa_{E}$. If this operator is not invertible, solutions of the posed inversion problem can be brought.

In the recent papers $[2,4]$, we study the inverse problem stability of the continuous-in-time model. We discuss this study with determining Loan Measure $\widetilde{\kappa}_{E}$ from Algebraic Spending Measure $\widetilde{\sigma}$ in Radon measure space, that is, $F=$ $\mathscr{M}\left(\left[t_{\mathrm{I}}, \Theta_{\max }-\Theta_{\gamma}\right]\right)$ and $G=\mathscr{M}\left(\left[t_{\mathrm{I}}, \Theta_{\max }\right]\right)$, and in Hilbert space, that is, $F=\mathbb{L}^{2}\left(\left[t_{\mathrm{I}}, \Theta_{\max }-\Theta_{\gamma}\right]\right)$ and $G=\mathbb{L}^{2}\left(\left[t_{\mathrm{I}}, \Theta_{\max }\right]\right)$, when they are density measures. For this inverse problem we prove the uniqueness theorem in [4]; we obtain a procedure 
for constructing the solution and provide necessary and sufficient conditions for the solvability of the inverse problem.

We are motivated by a recently developed nonlinear inverse scale in Schwartz space. We refer the reader to [5, 6], for applications of fast inversion formulas to inverse problems. Bauer and Lukas investigate in [5] some different frameworks for regularization of linear inverse problems when error is expected to be decreased at infinity. In the paper [6], Hansen investigates the approximation properties of regularized solutions to discrete ill-posed. They average decay to zero faster than the generalized singular values.

We show in this paper some results of this inverse problem in Schwartz space. We sketch the theoretical results that justify the mathematical well-posedness under some assumptions. The main result of this paper is to study the existence and uniqueness of solutions. We give an overview of properties for operator $\mathscr{L}$, describing the computation of its image.

The rest of this paper is arranged as follows. In Section 2 we introduce the definition of operator $\mathscr{L}$ and others, and the mathematical properties of these operators are shown. We treat in Section 3 the spectrum of some operators involved in the model by determining the inverse of operator under some hypothesis. It is followed by enrichment of the model of variable rate in Section 4. In Section 5, we examine the concept of ill-posedness in Schwartz space in order to obtain interesting and useful solutions.

\section{Properties of Operators}

This section is devoted to explore mathematical properties of some operators involved in the model. Those properties will be useful for some aspects of the model implementation to come in the following. These operators are acting on measures over $\mathbb{R}$. For that, we will also consider that $\mathscr{M}\left(\left[t_{\mathrm{I}}, \Theta_{\max }\right]\right)$ is the set of Radon Measures over $\mathbb{R}$, supported in $\left[t_{\mathrm{I}}, \Theta_{\text {max }}\right]$. In the sequel, we consider the case when all measures are density measures. The purpose is to compute the adjoint of these operators. We will be able to use some specific mathematical tools as inner product.

We proceed by denoting $\mathbb{L}^{2}\left(\left[t_{\mathrm{I}}, \Theta_{\max }\right]\right)$ the space of square-integrable functions over $\mathbb{R}$ having their support in $\left[t_{\mathrm{I}}, \Theta_{\max }\right]$. We state the Repayment Pattern Density $\gamma$ as follows:

$$
\gamma \in \mathbb{L}^{2}\left(\left[0, \Theta_{\gamma}\right]\right)
$$

where $\Theta_{\gamma}$ is a positive number such that

$$
\Theta_{\gamma}<\Theta_{\max }-t_{\mathrm{I}}
$$

We recall that we have shown the balanced equation given by equality (14) in [2]. This equality consists in writing Loan Density $\kappa_{E}$ as a sum of Algebraic Spending Density $\sigma$ and densities associated with quantities that have to be repaid or paid. This equality yields with convolution equality defined by (9) in [2] to express density $\sigma$ :

$$
\begin{aligned}
\sigma(t)= & \kappa_{E}(t)-\left(\kappa_{E} \star \gamma\right)(t)-\alpha \int_{t_{\mathrm{I}}}^{t}\left(\kappa_{E}-\kappa_{E} \star \gamma\right)(s) d s \\
& -\alpha \int_{t}^{\Theta_{\max }} \rho_{\mathscr{K}}^{\mathrm{I}}(s) d s-\rho_{\mathscr{K}}^{\mathrm{I}}(t) .
\end{aligned}
$$

From this, linear term of density $\sigma$ is defined by linear operator $\mathscr{L}$ acting on Loan Density $\kappa_{E} \in \mathbb{L}^{2}\left(\left[t_{\mathrm{I}}, \Theta_{\max }-\Theta_{\gamma}\right]\right)$ given by

$$
\begin{aligned}
\mathscr{L}\left[\kappa_{E}\right](t)= & \kappa_{E}(t)-\left(\kappa_{E} \star \gamma\right)(t) \\
& -\alpha \int_{t_{\mathrm{I}}}^{t}\left(\kappa_{E}-\kappa_{E} \star \gamma\right)(s) d s .
\end{aligned}
$$

The aim here is to compute operator $\mathscr{L}^{*}$. For that, we will compute inner product $\left\langle\mathscr{L}\left[\kappa_{E}\right], \kappa_{F}\right\rangle$ for any densities $\kappa_{E}$ and $\kappa_{F}$ in, respectively, spaces $\mathbb{L}^{2}\left(\left[t_{\mathrm{I}}, \Theta_{\max }-\Theta_{\gamma}\right]\right)$ and $\mathbb{L}^{2}\left(\left[t_{\mathrm{I}}, \Theta_{\max }\right]\right)$ defined by

$$
\begin{aligned}
& \left\langle\mathscr{L}\left[\kappa_{E}\right], \kappa_{F}\right\rangle=\int_{t_{\mathrm{I}}}^{\Theta_{\max }} \mathscr{L}\left[\kappa_{E}\right](x) \kappa_{F}(x) d x \\
& =\int_{t_{\mathrm{I}}}^{\Theta_{\max }} \kappa_{E}(x) \kappa_{F}(x) d x \\
& \quad-\int_{t_{\mathrm{I}}}^{\Theta_{\max }}\left(\kappa_{E} \star \gamma\right)(x) \kappa_{F}(x) d x \\
& -\alpha \int_{t_{\mathrm{I}}}^{\Theta_{\max }}\left(\int_{t_{\mathrm{I}}}^{x} \kappa_{E}(y) d y\right) \kappa_{F}(x) d x \\
& +\alpha \int_{t_{\mathrm{I}}}^{\Theta_{\max }}\left(\int_{t_{\mathrm{I}}}^{x} \kappa_{E} \star \gamma(y) d y\right) \kappa_{F}(x) d x .
\end{aligned}
$$

We will simplify inner product $\left\langle\mathscr{L}\left[\kappa_{E}\right], \kappa_{F}\right\rangle$ given by relation (8) in function of four terms. Since first term $\int_{t_{\mathrm{I}}}^{\Theta_{\max }} \kappa_{E}(x) \kappa_{F}(x) d x$ is already simplified, we simplify the second one as follows:

$$
\begin{aligned}
& \int_{t_{\mathrm{I}}}^{\Theta_{\max }}\left(\kappa_{E} \star \gamma\right)(x) \kappa_{F}(x) d x \\
& =\int_{t_{\mathrm{I}}}^{\Theta_{\max }}\left(\int_{t_{\mathrm{I}}}^{x} \gamma(x-y) \kappa_{E}(y) d y\right) \kappa_{F}(x) d x \\
& =\int_{t_{\mathrm{I}}}^{\Theta_{\max }}\left(\int_{t_{\mathrm{I}}}^{\Theta_{\max }} \gamma(x-y) \kappa_{E}(y) \mathbb{1}_{\{y \leq x\}} d y\right) \kappa_{F}(x) d x \\
& =\int_{t_{\mathrm{I}}}^{\Theta_{\max }} \kappa_{E}(y)\left(\int_{y}^{\Theta_{\max }} \gamma(x-y) \kappa_{F}(x) d x\right) d y .
\end{aligned}
$$


Next, we simplify the third one as follows:

$$
\begin{aligned}
& \int_{t_{\mathrm{I}}}^{\Theta_{\max }}\left(\int_{t_{\mathrm{I}}}^{x} \kappa_{E}(y) d y\right) \kappa_{F}(x) d x \\
& =\int_{t_{\mathrm{I}}}^{\Theta_{\max }}\left(\int_{t_{\mathrm{I}}}^{\Theta_{\max }} \kappa_{E}(y) \mathbb{1}_{\{y \leq x\}} d y\right) \kappa_{F}(x) d x \\
& =\int_{t_{\mathrm{I}}}^{\Theta_{\max }} \kappa_{E}(y)\left(\int_{y}^{\Theta_{\max }} \kappa_{F}(x) d x\right) d y .
\end{aligned}
$$

The last one is simplified as follows:

$$
\begin{aligned}
& \int_{t_{\mathrm{I}}}^{\Theta_{\max }}\left(\int_{t_{\mathrm{I}}}^{x} \kappa_{E} \star \gamma(y) d y\right) \kappa_{F}(x) d x \\
& =\int_{t_{\mathrm{I}}}^{\Theta_{\max }}\left(\int_{t_{\mathrm{I}}}^{x}\left(\int_{t_{\mathrm{I}}}^{y} \gamma(y-t) \kappa_{E}(t) d t\right) d y\right) \kappa_{F}(x) d x \\
& =\int_{t_{\mathrm{I}}}^{\Theta_{\max }}\left(\int_{t_{\mathrm{I}}}^{\Theta_{\max }} \mathbb{1}_{\{y \leq x\}}\left(\int_{t_{\mathrm{I}}}^{\Theta_{\max }} \gamma(y-t) \kappa_{E}(t) \mathbb{1}_{\{t \leq y\}} d t\right) d y\right) \\
& \cdot \cdot \kappa_{F}(x) d x \\
& =\int_{t_{\mathrm{I}}}^{\Theta_{\max }}\left(\int_{t_{\mathrm{I}}}^{\Theta_{\max }} \kappa_{E}(t) \mathbb{1}_{\{t \leq x\}}\left(\int_{t_{\mathrm{I}}}^{\Theta_{\max }} \gamma(y-t) \mathbb{1}_{\{t \leq y \leq x\}} d y\right) d t\right) \\
& \cdot \cdot \kappa_{F}(x) d x \\
& =\int_{t_{\mathrm{I}}}^{\Theta_{\max }}\left(\int_{t_{\mathrm{I}}}^{\Theta_{\max }} \kappa_{F}(x) \mathbb{1}_{\{t \leq x\}}\left(\int_{t_{\mathrm{I}}}^{\Theta_{\max }} \gamma(y-t) \mathbb{1}_{\{t \leq y \leq x\}} d y\right) d x\right) \\
& \cdot \cdot \kappa_{E}(t) d t .
\end{aligned}
$$

According to relations (9), (10), and (11), expression (8) of inner product $\left\langle\mathscr{L}\left[\kappa_{E}\right], \kappa_{F}\right\rangle$ reads

$$
\begin{aligned}
& \left\langle\mathscr{L}\left[\kappa_{E}\right], \kappa_{F}\right\rangle=\int_{t_{\mathrm{I}}}^{\Theta_{\max }} \kappa_{E}(y)\left(\kappa_{F}(y)\right. \\
& -\int_{y}^{\Theta_{\max }} \gamma(x-y) \kappa_{F}(x) d x-\alpha \int_{y}^{\Theta_{\max }} \kappa_{F}(x) d x \\
& \left.+\alpha \int_{y}^{\Theta_{\max }} \kappa_{F}(x)\left(\int_{y}^{x} \gamma(z-y) d z\right) d x\right) d y .
\end{aligned}
$$

Since operator $\mathscr{L}^{*}$ is one such that

$$
\begin{aligned}
& \left\langle\mathscr{L}\left[\kappa_{E}\right], \kappa_{F}\right\rangle=\left\langle\kappa_{E}, \mathscr{L}^{*}\left[\kappa_{F}\right]\right\rangle, \\
& \forall \kappa_{E} \in \mathbb{L}^{2}\left(\left[t_{\mathrm{I}}, \Theta_{\max }-\Theta_{\gamma}\right]\right), \forall \kappa_{F} \in \mathbb{L}^{2}\left(\left[t_{\mathrm{I}}, \Theta_{\max }\right]\right),
\end{aligned}
$$

and, according to relation (12), operator $\mathscr{L}^{*}$ is defined by

$$
\begin{gathered}
\mathscr{L}^{*}\left[\kappa_{F}\right](y)=\kappa_{F}(y)-\int_{y}^{\Theta_{\max }} \gamma(x-y) \kappa_{F}(x) d x \\
-\alpha \int_{y}^{\Theta_{\max }} \kappa_{F}(x) d x+\alpha \int_{y}^{\Theta_{\max }} \kappa_{F}(x)
\end{gathered}
$$

$$
\begin{aligned}
& \cdot\left(\int_{y}^{x} \gamma(z-y) d z\right) d x \\
= & \kappa_{F}(y)-\int_{y}^{\Theta_{\max }} \kappa_{F}(x) \\
& \cdot\left(\gamma(x-y)+\alpha-\alpha \int_{y}^{x} \gamma(z-y) d z\right) d x .
\end{aligned}
$$

Define linear operator $\mathscr{D}$ acting on Initial Debt Repayment Density $\rho_{\mathscr{K}}^{\mathrm{I}} \in \mathbb{L}^{2}\left(\left[t_{\mathrm{I}}, \Theta_{\max }\right]\right)$ as

$$
\mathscr{D}\left[\rho_{\mathscr{K}}^{\mathrm{I}}\right](t)=-\alpha \int_{t}^{\Theta_{\max }} \rho_{\mathscr{K}}^{\mathrm{I}}(s) d s-\rho_{\mathscr{K}}^{\mathrm{I}}(t)
$$

The integration by parts states that inner product $\left\langle\mathscr{D}\left[\rho_{\mathscr{K}}^{\mathrm{I}}\right]\right.$, $\left.\kappa_{F}\right\rangle$ is computed for any densities $\rho_{\mathscr{K}}^{I}$ and $\kappa_{F}$ in $\mathbb{L}^{2}\left(\left[t_{\mathrm{I}}, \Theta_{\max }\right]\right)$ as follows:

$$
\begin{aligned}
& \left\langle\mathscr{D}\left[\rho_{\mathscr{K}}^{\mathrm{I}}\right], \kappa_{F}\right\rangle=\int_{t_{\mathrm{I}}}^{\Theta_{\max }} \mathscr{D}\left[\rho_{\mathscr{K}}^{\mathrm{I}}\right](x) \kappa_{F}(x) d x \\
& =-\alpha \int_{t_{\mathrm{I}}}^{\Theta_{\max }}\left(\int_{x}^{\Theta_{\max }} \rho_{\mathscr{K}}^{\mathrm{I}}(y) d y\right) \kappa_{F}(x) d x \\
& \quad-\int_{t_{\mathrm{I}}}^{\Theta_{\max }} \rho_{\mathscr{K}}^{\mathrm{I}}(x) \kappa_{F}(x) d x \\
& =\alpha \int_{t_{\mathrm{I}}}^{\Theta_{\max }} \rho_{\mathscr{K}}^{\mathrm{I}}(x)\left(\int_{x}^{\Theta_{\max }} \kappa_{F}(y) d y\right) d x \\
& +\alpha\left(\int_{t_{\mathrm{I}}}^{\Theta_{\max }} \rho_{\mathscr{K}}^{\mathrm{I}}(y) d y\right)\left(\int_{t_{\mathrm{I}}}^{\Theta_{\max }} \kappa_{F}(y) d y\right) \\
& \quad-\int_{t_{\mathrm{I}}}^{\Theta_{\max }} \rho_{\mathscr{K}}^{\mathrm{I}}(x) \kappa_{F}(x) d x \\
& =\int_{t_{\mathrm{I}}}^{\Theta_{\max }} \rho_{\mathscr{K}}^{\mathrm{I}}(x)\left(\alpha \int_{x}^{\Theta_{\max }} \kappa_{F}(y) d y\right. \\
& \left.\quad+\alpha \int_{t_{\mathrm{I}}}^{\Theta_{\max }} \kappa_{F}(y) d y-\kappa_{F}(x)\right) d x .
\end{aligned}
$$

From this, operator $\mathscr{D}^{*}$ is defined by

$$
\begin{aligned}
\mathscr{D}^{*}\left[\kappa_{F}\right](x)= & \alpha \int_{x}^{\Theta_{\max }} \kappa_{F}(y) d y+\alpha \int_{t_{\mathrm{I}}}^{\Theta_{\max }} \kappa_{F}(y) d y \\
& -\kappa_{F}(x) .
\end{aligned}
$$

Operator $\mathscr{L}$ we set out in relation (7) considered a constant rate. Nevertheless, if we consider in it a function $\alpha$ that depends on $t$, the model becomes a financial model with 
variable rate. The only modification to make is to enrich (7) and (15) by writing

$$
\begin{aligned}
\mathscr{L}\left[\kappa_{E}\right](t)= & \kappa_{E}(t)-\left(\kappa_{E} \star \gamma\right)(t) \\
& -\alpha(t) \int_{t_{\mathrm{I}}}^{t}\left(\kappa_{E}-\kappa_{E} \star \gamma\right)(s) d s . \\
\mathscr{D}\left[\rho_{\mathscr{K}}^{\mathrm{I}}\right](t)= & -\alpha(t) \int_{t}^{\Theta_{\max }} \rho_{\mathscr{K}}^{\mathrm{I}}(s) d s-\rho_{\mathscr{K}}^{\mathrm{I}}(t) .
\end{aligned}
$$

Once this enrichment is done, using a variable rate makes operator $\mathscr{D}^{*}$ expressed in terms of densities $\alpha$ and $\gamma$ :

$$
\begin{aligned}
& \mathscr{L}^{*}\left[\kappa_{F}\right](y)=\kappa_{F}(y)-\int_{y}^{\Theta_{\max }} \kappa_{F}(x)(\gamma(x-y) \\
& \left.+\alpha(x)-\alpha(x) \int_{y}^{x} \gamma(z-y) d z\right) d x .
\end{aligned}
$$

By definition $\alpha(x)$ is the rate at time $x$. Operator $\mathscr{D}^{*}$ given by relation (17) can be rewritten adding this enrichment

$$
\begin{aligned}
\mathscr{D}^{*}\left[\kappa_{F}\right](x)= & \alpha(x) \int_{x}^{\Theta_{\max }} \kappa_{F}(y) d y \\
& +\alpha(x) \int_{t_{\mathrm{I}}}^{\Theta_{\max }} \kappa_{F}(y) d y-\kappa_{F}(x) .
\end{aligned}
$$

Lemma 1. The image of operator $\mathscr{L}$ is such that

$$
\operatorname{Im}(\mathscr{L})^{\perp}=\{0\} .
$$

Proof. In order to show equality (22), we will show that the kernel of operator $\mathscr{L}^{*}$ is reduced to null set because of the following property:

$$
\operatorname{Im}(\mathscr{L})^{\perp}=\operatorname{Ker}\left(\mathscr{L}^{*}\right)
$$

According to (14), if density $\kappa_{F}$ is in $\operatorname{Ker}\left(\mathscr{L}^{*}\right)$, then, we get the following equation:

$$
\begin{aligned}
& \kappa_{F}(y)-\int_{y}^{\Theta_{\max }} \kappa_{F}(x) \\
& \quad\left(\gamma(x-y)+\alpha-\alpha \int_{y}^{x} \gamma(z-y) d z\right) d x=0 .
\end{aligned}
$$

Deriving, we get the ODE that the solution $\kappa_{F}$ is expressed as follows:

$$
\kappa_{F}^{\prime}(y)-(\alpha+\gamma(0)) \kappa_{F}(y)=0 .
$$

The general solution to (25) is given by

$$
\kappa_{F}(y)=\kappa_{F}\left(t_{\mathrm{I}}\right) e^{-(\alpha+\gamma(0))\left(y-t_{\mathrm{I}}\right)},
$$

where initial condition $\kappa_{F}\left(t_{\mathrm{I}}\right)$ stands for the value of density $\kappa_{F}$ at initial time $t_{\mathrm{I}}$. On the other hand, (24) is equivalent to

$$
\begin{aligned}
& \underbrace{\kappa_{F}(y)-(\alpha+\gamma(0)) \int_{\max }^{\Theta_{\max }} \kappa_{F}(x) d x}_{\text {First term } I_{1}} \\
& +\underbrace{\int_{y}^{\Theta_{\max }}\left(-\gamma(x-y)+\gamma(0)+\alpha \int_{y}^{x} \gamma(z-y) d z\right) d x}_{\text {Second term } I_{2}}
\end{aligned}
$$

$=0$.

Conversely, we will show that density $\kappa_{F}$ is zero. In the first place, replacing density $\kappa_{F}$ given by (26) in first term $I_{1}$ of (27), we get

$$
\begin{gathered}
\kappa_{F}(y)-(\alpha+\gamma(0)) \int_{y}^{\Theta_{\max }} \kappa_{F}(x) d x \\
=\frac{-\alpha \kappa_{F}\left(t_{\mathrm{I}}\right)}{\alpha+\gamma(0)} e^{-(\alpha+\gamma(0))\left(\Theta_{\max }-t_{\mathrm{I}}\right)} .
\end{gathered}
$$

Secondly, the second term $I_{2}$ is a constant function due to its derivative which equals zero:

$$
\begin{aligned}
& \left(\int_{y}^{\Theta_{\max }}(-\gamma(x-y)+\gamma(0)\right. \\
& \left.\left.\quad+\alpha \int_{y}^{x} \gamma(z-y) d z\right) d x\right)^{\prime}=0-(-\gamma(0) \\
& \left.\quad+\gamma(0)+\alpha \int_{y}^{y} \gamma(z-y) d z\right) \\
& =0 .
\end{aligned}
$$

Consequently, the second term $I_{2}$ is equal to a real constant $C$ to be determined:

$$
\begin{aligned}
& \int_{y}^{\Theta_{\max }}\left(-\gamma(x-y)+\gamma(0)+\alpha \int_{y}^{x} \gamma(z-y) d z\right) d x \\
& \quad=C .
\end{aligned}
$$

The initial condition is obtained from integral equation defined by (30) with replacing $y$ by $\Theta_{\max }$, which implies that constant $C$ is zero. It is concluded that

$$
\begin{aligned}
& \int_{y}^{\Theta_{\max }}\left(-\gamma(x-y)+\gamma(0)+\alpha \int_{y}^{x} \gamma(z-y) d z\right) d x \\
& \quad=0, \quad \forall y \in\left[t_{\mathrm{I}}, \Theta_{\max }\right] .
\end{aligned}
$$

Then, relations (27), (28), and (31) yield the following equality:

$$
\frac{-\alpha \kappa_{F}\left(t_{\mathrm{I}}\right)}{\alpha+\gamma(0)} e^{-(\alpha+\gamma(0))\left(\Theta_{\max }-t_{\mathrm{I}}\right)}=0
$$

From this, initial density $\kappa_{F}\left(t_{\mathrm{I}}\right)$ or loan rate $\alpha$ is zero since exponential function is positive. It follows that if $\kappa_{F}\left(t_{\mathrm{I}}\right)$ is 
zero, then, density $\kappa_{F}$ is zero, which is obtained from relation (26). In this case:

$$
\operatorname{Ker}\left(\mathscr{L}^{*}\right)=\{0\}
$$

If loan rate $\alpha$ is zero, then, according to (24), we obtain following integral equation:

$$
\kappa_{F}(y)-\int_{y}^{\Theta_{\max }} \kappa_{F}(x) \gamma(x-y) d x=0
$$

where expression of density $\kappa_{F}$ is determined from equality (26) as

$$
\kappa_{F}(y)=\kappa_{F}\left(t_{\mathrm{I}}\right) e^{-\gamma(0)\left(y-t_{\mathrm{I}}\right)} .
$$

If density $\kappa_{F}$ given by (35) is coupled with (34), then, $\kappa_{F}\left(t_{\mathrm{I}}\right)$ is zero allowing zero density $\kappa_{F}$. We showed that density $\kappa_{F}$ is zero in both cases. From this, we can deduce that (33) is true, proving the lemma.

\section{Spectrum of Operators}

It is well known that the integral operators $[7,8]$ possess a very rich structure theory, such that these operators played an important role in the study of operators on Hilbert Spaces. The paper [9] and book [10] by Gil' deal with the spectra of a class of linear non-self-adjoint operators containing the Volterra operators. Since this operator is involved in the model, we use it in order to study the spectrum of some operators. It is shown in [11] that the spectrum of Volterra composition operator is consisting of zero only.

This section is devoted to explore the spectrum of some operators involving the spectrum of Volterra. Defining linear operator $\widetilde{\mathscr{V}}: \mathbb{L}^{2}\left(\left[t_{\mathrm{I}}, \Theta_{\max }-\Theta_{\gamma}\right]\right) \rightarrow \mathbb{L}^{2}\left(\left[t_{\mathrm{I}}, \Theta_{\max }\right]\right)$ by operator that is acting on Loan Density $\kappa_{E}$,

$$
\widetilde{\mathscr{V}}\left[\kappa_{E}\right](t)=\kappa_{E} \star \gamma(t)+\alpha \int_{t_{\mathrm{I}}}^{t}\left(\kappa_{E}-\kappa_{E} \star \gamma\right)(s) d s .
$$

The canonical injection $\operatorname{Id}_{\mathbb{L}^{2}\left(\left[t_{\mathbb{I}}, \Theta_{\max }-\Theta_{\gamma}\right]\right) \rightarrow \mathbb{L}^{2}\left(\left[t_{\mathbb{I}}, \Theta_{\max }\right]\right)}$ is defined from $\mathbb{L}^{2}\left(\left[t_{\mathrm{I}}, \Theta_{\max }-\Theta_{\gamma}\right]\right)$ to $\mathbb{L}^{2}\left(\left[t_{\mathrm{I}}, \Theta_{\max }\right]\right)$ as

$$
\operatorname{Id}_{\mathbb{L}^{2}\left(\left[t_{I}, \Theta_{\max }-\Theta_{\gamma}\right]\right) \rightarrow \mathbb{L}^{2}\left(\left[t_{I}, \Theta_{\max }\right]\right)}\left[\kappa_{E}\right](t)=\left[\kappa_{E}\right](t),
$$

which is decomposed as a sum of operators $\mathscr{L}$ and $\widetilde{\mathscr{V}}$ given by relations (7) and (36), respectively:

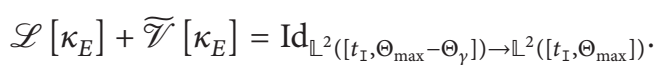

Theorem 2. If density $\gamma$ has upper bound $M-2|\alpha|$ over its support:

$$
\sup _{z \in\left[0, \Theta_{\gamma}\right]}\{|\gamma(z)|\}=M-2|\alpha|
$$

where $M$ is a positive real satisfying

$$
2|\alpha|<M<\frac{1}{\Theta_{\max }-t_{\mathrm{I}}} ;
$$

then, operator $\mathscr{L}$ is invertible, where its inverse $\mathscr{L}^{-1}$ is given by

$$
\mathscr{L}^{-1}\left[\kappa_{E}\right](x)=J \circ\left(\sum_{k \geq 0}(\widetilde{\mathscr{V}} \circ J)^{(k)}\right)\left[\kappa_{E}\right](x),
$$

where $J$ is an operator defined by

$$
\begin{aligned}
& J: \mathbb{L}^{2}\left(\left[t_{\mathrm{I}}, \Theta_{\max }\right]\right) \longrightarrow \mathbb{L}^{2}\left(\left[t_{\mathrm{I}}, \Theta_{\max }-\Theta_{\gamma}\right]\right) \\
& \kappa_{E} \quad \longmapsto \quad \kappa_{E}
\end{aligned}
$$

Proof. Since operator $\widetilde{\mathscr{V}}$ is defined from $\mathbb{L}^{2}\left(\left[t_{\mathrm{I}}, \Theta_{\max }-\Theta_{\gamma}\right]\right)$ to $\mathbb{L}^{2}\left(\left[t_{\mathrm{I}}, \Theta_{\max }\right]\right)$ and by using definition (42) of operator $J$, we get

$$
\widetilde{\mathscr{V}} \circ J\left(\mathbb{L}^{2}\left(\left[t_{\mathrm{I}}, \Theta_{\max }\right]\right)\right) \subset \mathbb{L}^{2}\left(\left[t_{\mathrm{I}}, \Theta_{\max }\right]\right) .
$$

From this, we get

$$
(\widetilde{\mathscr{V}} \circ J)^{(k)}\left(\mathbb{L}^{2}\left(\left[t_{\mathrm{I}}, \Theta_{\max }\right]\right)\right) \subset \mathbb{L}^{2}\left(\left[t_{\mathrm{I}}, \Theta_{\max }\right]\right),
$$

$\forall k \in \mathbb{N}$,

which implies that

$$
\begin{gathered}
J \circ\left(\sum_{k \geq 0}(\widetilde{\mathscr{V}} \circ J)^{(k)}\right)\left(\mathbb{L}^{2}\left(\left[t_{\mathrm{I}}, \Theta_{\max }\right]\right)\right) \\
\subset \mathbb{L}^{2}\left(\left[t_{\mathrm{I}}, \Theta_{\max }-\Theta_{\gamma}\right]\right) .
\end{gathered}
$$

Relation (45) shows that equality (41) is consistent due to the inverse of operator $\mathscr{L}$ being in $\mathbb{L}^{2}\left(\left[t_{\mathrm{I}}, \Theta_{\max }-\Theta_{\gamma}\right]\right)$. Next, we can show that operator $\widetilde{\mathscr{V}} \circ J$ can be written in the form

$$
\widetilde{\mathscr{V}} \circ J\left[\kappa_{E}\right](x)=\int_{t_{\mathrm{I}}}^{x} k(x, y) \kappa_{E}(y) d y,
$$

where kernel $k$ is defined as

$$
k(x, y)=\gamma(x-y)+\alpha-\alpha \int_{y}^{x} \gamma(t-y) d t .
$$

Following equality

$$
\begin{aligned}
& \left\{(y, t) \in \mathbb{R}^{2} / t_{\text {I }} \leq y \leq t, t_{\text {I }} \leq t \leq x\right\} \\
& \quad=\left\{(y, t) \in \mathbb{R}^{2} / t_{\text {I }} \leq y \leq x, y \leq t \leq x\right\}
\end{aligned}
$$

yields with Fubini-Tonelli theorem to obtain

$$
\begin{aligned}
& (\widetilde{\mathscr{V}} \circ J)^{(2)}\left[\kappa_{E}\right](x)=\int_{t_{\mathrm{I}}}^{x} k(x, t)(\widetilde{\mathscr{V}} \circ J)\left[\kappa_{E}\right](t) d t \\
& =\int_{t_{\mathrm{I}}}^{x} k(x, t)\left(\int_{t_{\mathrm{I}}}^{t} k(t, y) \kappa_{E}(y) d y\right) d t \\
& =\int_{t_{\mathrm{I}}}^{x}\left(\int_{y}^{x} k(x, t) k(t, y) d t\right) \kappa_{E}(y) d y .
\end{aligned}
$$


Consequently, operator $(\widetilde{\mathscr{V}} \circ J)^{(2)}$ is written in following form:

$$
(\widetilde{\mathscr{V}} \circ J)^{(2)}\left[\kappa_{E}\right](x)=\int_{t_{\mathrm{I}}}^{x} k_{2}(x, y) \kappa_{E}(y) d y,
$$

where

$$
k_{2}(x, y)=\int_{y}^{x} k(x, t) k(t, y) d t .
$$

We can verify by induction for $n \geq 2$ that the recurrence expresses each operator $(\widetilde{\mathscr{V}} \circ J)^{(n)}$ as an integral operator which is written in following form:

$$
(\widetilde{\mathscr{V}} \circ J)^{(n)}\left[\kappa_{E}\right](x)=\int_{t_{\mathrm{I}}}^{x} k_{n}(x, y) \kappa_{E}(y) d y,
$$

where kernel $k_{n}$ is given as

$$
k_{n}(x, y)=\int_{y}^{x} k(x, t) k_{n-1}(t, y) d t
$$

Now, we will show that $M$ is a maximum of kernel $k$ over $\left[t_{\mathrm{I}}, \Theta_{\max }\right] \times\left[t_{\mathrm{I}}, \Theta_{\max }-\Theta_{\gamma}\right]$ with using equality (39):

$$
\begin{aligned}
|k(x, y)| & \leq|\gamma(x-y)|+|\alpha|\left|\int_{0}^{\Theta_{\gamma}} \gamma(z) d t\right|+|\alpha| \\
& \leq M-2|\alpha|+|\alpha|+|\alpha| \\
& \leq M .
\end{aligned}
$$

By a recurrence starting with initial state $n=1$ being true, it is easy to prove that

$$
\left|k_{n}(x, y)\right| \leq M^{n} \frac{(x-y)^{n-1}}{(n-1) !} .
$$

If $n=1$, inequality (55) is true because $M$ is the maximum of the kernel $k$ over $\left[t_{\mathrm{I}}, \Theta_{\max }\right] \times\left[t_{\mathrm{I}}, \Theta_{\max }-\Theta_{\gamma}\right]$. Assume that inequality (55) is true for a case $n$ and show a case $n+1$. According to (53), we get

$$
\begin{aligned}
\left|k_{n+1}(x, y)\right| & \leq \int_{y}^{x}|k(x, t)|\left|k_{n}(t, y)\right| d t \\
& \leq M \times \frac{M^{n}}{(n-1) !} \int_{y}^{x}(t-y)^{n-1} d t \\
& \leq M \times \frac{M^{n}}{(n-1) !} \times \frac{(x-y)^{n}}{n} \\
& \leq M^{n+1} \frac{(x-y)^{n}}{n !} .
\end{aligned}
$$

Since $x-y \in\left[0, \Theta_{\gamma}\right]$, we use inequality (55) to get

$$
\left|k_{n}(x, y)\right| \leq M^{n} \frac{\Theta_{\gamma}^{n-1}}{(n-1) !} .
$$

Applying Cauchy-Schwarz inequality at $(\widetilde{\mathscr{V}} \circ J)^{(n)}$ defined by (52), we get following equality:

$$
\begin{aligned}
& \left|(\widetilde{\mathscr{V}} \circ J)^{(n)}\left[\kappa_{E}\right](x)\right|^{2} \\
& \quad \leq\left(\int_{t_{\mathrm{I}}}^{x}\left|k_{n}(x, y)\right|^{2} d y\right)\left\|\kappa_{E}\right\|_{\mathbb{L}^{2}\left(\left[t_{\mathrm{I}}, \Theta_{\max }-\Theta_{\gamma}\right]\right)}^{2} .
\end{aligned}
$$

From this and using inequality (57), we get

$$
\begin{aligned}
& \left|(\widetilde{\mathscr{V}} \circ J)^{(n)}\left[\kappa_{E}\right](x)\right|^{2} \leq\left(\frac{M^{n}}{(n-1) !}\right)^{2} \\
& \quad \cdot\left(\int_{t_{\mathrm{I}}}^{x}(x-y)^{2(n-1)} d y\right)\left\|\kappa_{E}\right\|_{\mathbb{L}^{2}\left(\left[t_{\mathrm{I}}, \Theta_{\max }-\Theta_{\gamma}\right]\right)}^{2} \\
& \leq\left(\frac{M^{n}}{(n-1) !}\right)^{2} \times \frac{\left(x-t_{\mathrm{I}}\right)^{2 n-1}}{2 n-1}\left\|\kappa_{E}\right\|_{\mathbb{L}^{2}\left(\left[t_{\mathrm{I}}, \Theta_{\max }-\Theta_{\gamma}\right]\right)} .
\end{aligned}
$$

By integrating each term of (60) over interval $\left[t_{\mathrm{I}}, \Theta_{\max }\right]$, we get

$$
\left\|(\widetilde{\mathscr{V}} \circ J)^{(n)}\left[\kappa_{E}\right]\right\|_{\mathbb{L}^{2}\left(\left[t_{\mathrm{I}}, \Theta_{\max }\right]\right)}^{2} \leq\left(\frac{M^{n}}{(n-1) !}\right)^{2} \times \frac{\left(\Theta_{\max }-t_{\mathrm{I}}\right)^{2 n}}{2 n(2 n-1)}\left\|\kappa_{E}\right\|_{\mathbb{L}^{2}\left(\left[t_{\mathrm{I}}, \Theta_{\max }-\Theta_{\gamma}\right]\right)}^{2} .
$$

Consequently,

$$
\left\|(\widetilde{\mathscr{V}} \circ J)^{(n)}\right\|_{\mathbb{L}^{2}\left(\left[t_{\mathrm{I}}, \Theta_{\max }\right]\right)} \leq\left(\frac{M^{n}}{(n-1) !}\right) \times \frac{\left(\Theta_{\max }-t_{I}\right)^{n}}{\sqrt{2 n}} .
$$

Inequality (61) gives

$$
\begin{aligned}
& \left\|(\widetilde{\mathscr{V}} \circ J)^{(n)}\right\|_{\mathbb{L}^{2}\left(\left[t_{\mathrm{I}}, \Theta_{\max }\right]\right)}^{1 / n} \\
& \quad \leq M\left(\Theta_{\max }-t_{\mathrm{I}}\right) \times(\sqrt{2 n}(n-1) !)^{-1 / n} .
\end{aligned}
$$

Since the series $\sum_{k \geq 1} a_{k}$ of defined terms

$$
a_{k}=\frac{\left(M\left(\Theta_{\max }-t_{\mathrm{I}}\right)\right)^{k}}{\sqrt{2 k}(k-1) !}
$$

is convergent, then quantity $\sum_{k \geq 0}(\widetilde{\mathscr{V}} \circ J)^{(k)}$ converges absolutely in $\mathbb{L}^{2}\left(\left[t_{\mathrm{I}}, \Theta_{\max }\right]\right)$. Consequently, $\sum_{k \geq 0}(\widetilde{\mathscr{V}} \circ J)^{(k)}$ exists and is finite in $\mathbb{L}^{2}\left(\left[t_{\mathrm{I}}, \Theta_{\max }\right]\right)$. We recall that we have

$$
\left(\operatorname{Id}_{\mathbb{L}^{2}\left(\left[t_{\mathrm{I}}, \Theta_{\max }\right]\right)}-\widetilde{\mathscr{V}} \circ J\right) \circ\left(\sum_{k=0}^{N}(\widetilde{\mathscr{V}} \circ J)^{(k)}\right)\left[\kappa_{E}\right](x)
$$




$$
\begin{aligned}
= & \sum_{k=0}^{N}(\widetilde{\mathscr{V}} \circ J)^{(k)}\left[\kappa_{E}\right](x) \\
& -\sum_{k=0}^{N}(\widetilde{\mathscr{V}} \circ J)^{(k+1)}\left[\kappa_{E}\right](x) \\
= & \operatorname{Id}_{\mathbb{L}^{2}\left(\left[t_{\mathrm{I}}, \Theta_{\max }\right]\right)}\left[\kappa_{E}\right](x)-\widetilde{\mathscr{V}}^{(N+1)}\left[\kappa_{E}\right](x) .
\end{aligned}
$$

Since we have $\sqrt{n} \leq(n-1)$ ! for all integer $n \geq 2$, inequality (61) implies that

$$
\left\|(\widetilde{\mathscr{V}} \circ J)^{(n)}\right\|_{\mathbb{L}^{2}\left(\left[t_{\mathrm{I}}, \Theta_{\max }\right]\right)} \leq\left(\frac{\left(M\left(\Theta_{\max }-t_{\mathrm{I}}\right)\right)^{n}}{\sqrt{2} n}\right) .
$$

Coupling inequality (64) with the fact that $\|(\widetilde{\mathscr{V}}$. $J)^{(n)} \|_{\mathbb{L}^{2}\left(\left[t_{\mathbb{I}}, \Theta_{\max }\right]\right)}$ converges to 0 due to (40), we get

$$
\begin{aligned}
& \left(\operatorname{Id}_{\mathbb{L}^{2}\left(\left[t_{\mathrm{I}}, \Theta_{\max }\right]\right)}-\widetilde{\mathscr{V}} \circ J\right) \circ\left(\sum_{k \geq 0}(\widetilde{\mathscr{V}} \circ J)^{(k)}\right)\left[\kappa_{E}\right](x) \\
& \quad=\operatorname{Id}_{\mathbb{L}^{2}\left(\left[t_{\mathrm{I}}, \Theta_{\max }\right]\right)}\left[\kappa_{E}\right](x) .
\end{aligned}
$$

Composing operators defined by (38) with operator $J$, we get

$$
\begin{aligned}
\mathscr{L} \circ J\left[\kappa_{E}\right](x)= & \operatorname{Id}_{\mathbb{L}^{2}\left(\left[t_{\mathrm{I}}, \Theta_{\max }-\Theta_{\gamma}\right]\right) \rightarrow \mathbb{L}^{2}\left(\left[t_{\mathrm{I}}, \Theta_{\max }\right]\right)} \\
& \circ J\left[\kappa_{E}\right](x)-\widetilde{\mathscr{V}} \circ J\left[\kappa_{E}\right](x) \\
= & \operatorname{Id}_{\mathbb{L}^{2}\left(\left[t_{\mathrm{I}}, \Theta_{\max }\right]\right)}\left[\kappa_{E}\right](x)-\widetilde{\mathscr{V}} \\
& \circ J\left[\kappa_{E}\right](x) .
\end{aligned}
$$

According to (66) and (67), we get

$$
\begin{aligned}
& (\mathscr{L} \circ J) \circ\left(\sum_{k \geq 0}(\widetilde{\mathscr{V}} \circ J)^{(k)}\right)\left[\kappa_{E}\right](x) \\
& =\operatorname{Id}_{\mathbb{L}^{2}\left(\left[t_{\mathrm{I}}, \Theta_{\max }\right]\right)}\left[\kappa_{E}\right](x) .
\end{aligned}
$$

That is,

$$
\begin{aligned}
\mathscr{L} & \circ\left(J \circ\left(\sum_{k \geq 0}(\widetilde{\mathscr{V}} \circ J)^{(k)}\right)\right)\left[\kappa_{E}\right](x) \\
& =\operatorname{Id}_{\mathbb{L}^{2}\left(\left[t_{\mathrm{I}}, \Theta_{\max }\right]\right)}\left[\kappa_{E}\right](x),
\end{aligned}
$$

achieving equality (41) of the lemma.

Let us characterize under assumption of Theorem 2 a spectrum set $\operatorname{Sp}(\widetilde{\mathscr{V}} \circ J)$ of operator $\widetilde{\mathscr{V}} \circ J$. Defining spectrum $\operatorname{Sp}(\widetilde{\mathscr{V}} \circ J)$ as a set of reals $\mu$ such that $\widetilde{\mathscr{V}} \circ J-\mu \mathrm{Id}_{\mathbb{L}^{2}\left(\left[t_{\mathrm{I}}, \Theta_{\max }\right]\right)}$ is not invertible. Formally:

$$
\begin{aligned}
& \operatorname{Sp}(\widetilde{\mathscr{V}} \circ J) \\
& =\left\{\mu \in \mathbb{R}: \widetilde{\mathscr{V}} \circ J-\mu \operatorname{Id}_{\mathbb{L}^{2}\left(\left[t_{\mathbb{I}}, \Theta_{\max }\right]\right)} \text { is not invertible }\right\} .
\end{aligned}
$$

Defining spectral radius $\rho(\widetilde{\mathscr{V}} \circ J)$ of operator $\widetilde{\mathscr{V}} \circ J$ as

$$
\rho(\widetilde{\mathscr{V}} \circ J)=\{|\lambda|: \lambda \in \operatorname{Sp}(\widetilde{\mathscr{V}} \circ J)\}
$$

Note that spectrum $\mathrm{Sp}(\widetilde{\mathscr{V}} \circ J)$ is included in a disk of center 0 and radius $\|\widetilde{\mathscr{V}} \circ J\|_{\mathbb{L}^{2}\left(\left[t_{I}, \Theta_{\max }\right]\right)}$. That is to say

$$
\rho(\widetilde{\mathscr{V}} \circ J) \leq\|\widetilde{\mathscr{V}} \circ J\|_{\mathbb{L}^{2}\left(\left[t_{\mathrm{I}}, \Theta_{\max }\right]\right)}
$$

Now we will compute spectral radius $\rho(\widetilde{\mathscr{V}} \circ J)$ of operator $\widetilde{\mathscr{V}} \circ J$ using following equality:

$$
\rho(\widetilde{\mathscr{V}} \circ J)=\lim _{n \rightarrow+\infty}\left\|(\widetilde{\mathscr{V}} \circ J)^{(n)}\right\|_{\mathbb{L}^{2}\left(\left[t_{\mathrm{I}}, \Theta_{\max }\right]\right)}^{1 / n} .
$$

Since we have shown that $\left\|(\widetilde{\mathscr{V}} \circ J)^{(n)}\right\|_{\mathbb{L}^{2}\left(\left[t_{I}, \Theta_{\max }\right]\right)}^{1 / n}$ converges to 0 , equality $(73)$ shows that spectral radius $\rho(\widetilde{\mathscr{V}} \circ J)$ is zero:

$$
\rho(\widetilde{\mathscr{V}} \circ J)=0
$$

Consequently, spectrum $\operatorname{Sp}(\widetilde{\mathscr{V}} \circ J)$ is reduced to null set:

$$
\operatorname{Sp}(\widetilde{\mathscr{V}} \circ J)=\{0\} .
$$

\section{Extensions in the Model of Variable Rate}

We built in [2] the financial models that are used on simplified problems in order to show how they can be used in reality. This section is devoted to enrich the model in order to account for this reality. In particular, we will express the Algebraic Spending Density $\sigma_{\mathscr{I}}$ in the model with variable rate. The mathematical consistency of this density $\sigma_{\mathscr{I}}$ is analyzed.

The Current Debt Field $\mathscr{K}_{\mathrm{RD}}$ is related to Loan Measure $\widetilde{\kappa}_{E}$ and Repayment Measure $\widetilde{\rho}_{\mathscr{K}}$ by the following Ordinary Differential Equation:

$$
\frac{d \mathscr{K}_{\mathrm{RD}}}{d t}=\kappa_{E}(t)-\rho_{\mathscr{K}}(t)-\rho_{\mathscr{K}}^{\mathrm{I}}(t) .
$$

The solution of this ODE is expressed as follows:

$$
\begin{aligned}
\mathscr{K}_{\mathrm{RD}}(t)= & \mathscr{K}_{\mathrm{RD}}\left(t_{\mathrm{I}}\right)+\int_{t_{\mathrm{I}}}^{t} \kappa_{E}(s) d s-\int_{t_{\mathrm{I}}}^{t} \rho_{\mathscr{K}}(s) d s \\
& -\int_{t_{\mathrm{I}}}^{t} \rho_{\mathscr{K}}^{\mathrm{I}}(s) d s \\
= & \int_{t_{\mathrm{I}}}^{t} \kappa_{E}(s) d s-\int_{t_{\mathrm{I}}}^{t} \rho_{\mathscr{K}}^{\mathrm{I}}(s) d s \\
& +\int_{t}^{\Theta_{\max }} \rho_{\mathscr{K}}^{\mathrm{I}}(s) d s .
\end{aligned}
$$

Since the Interest Payment Density $\rho_{\mathscr{I}}$ is related to the Current Debt Field by a proportionality relation:

$$
\rho_{\mathscr{I}}(t)=\alpha(t) \mathscr{K}_{\mathrm{RD}}(t)
$$


the Interest Payment Density $\rho_{\mathscr{Y}}$ can be expressed in terms of Loan Density $\kappa_{E}$ :

$$
\begin{aligned}
\rho_{\mathscr{F}}(t)= & \alpha(t) \int_{t_{\mathrm{I}}}^{t} \kappa_{E}(s) d s-\alpha(t) \int_{t_{\mathrm{I}}}^{t}\left(\kappa_{E} \star \gamma\right)(s) d s \\
& +\alpha(t) \int_{t}^{\Theta_{\max }} \rho_{\mathscr{K}}^{\mathrm{I}}(s) d s .
\end{aligned}
$$

Since Density $\sigma$ reads

$$
\sigma(t)=\mathscr{L}\left[\kappa_{E}\right](t)+\mathscr{D}\left[\rho_{\mathscr{K}}^{\mathrm{I}}\right](t),
$$

where operators $\mathscr{L}$ and $\mathscr{D}$ are defined in relations (18) and (19), respectively, we get equality (6). From this, we get the following Ordinary Differential Equation:

$$
\sigma(t)=\frac{d \mathscr{K}_{\mathrm{RD}}(t)}{d t}-\rho_{\mathscr{F}}(t) .
$$

Defining $k_{\mathrm{RD}}(t, s)$ the Current Debt at time $t$ which is associated with the amount borrowed at time $s . k_{\mathrm{RD}}$ is related to Loan Density $\kappa_{E}$ using Repayment Pattern Density $\gamma$ by the following Ordinary Differential Equation:

$$
\frac{d k_{\mathrm{RD}}(t, s)}{d t}=-\gamma(t-s) \kappa_{E}(s),
$$

with initial condition $k_{\mathrm{RD}}(s, s)=\kappa_{E}(s)$ that expresses that The Current Debt at time $s$ which is related to the borrowed amount at time $s$ is the borrowed amount at time $s$. The solution of this differential equation is expressed as

$$
k_{\mathrm{RD}}(t, s)=\kappa_{E}(s)-\int_{s}^{t} \gamma(y-s) \kappa_{E}(s) d y .
$$

We will use the expression (81) of density $\sigma$ in order to express Algebraic Spending Density $\sigma_{\mathscr{I}}$ in the model with variable rate. Quantity $\sigma_{\mathscr{f}}(t, s)$ is defined such that the difference between spending and income at time $t$ is associated with the amount borrowed at time $s$. It is a time density with respect to both variables $s$ and $t$. Relation (81) yields

$$
\begin{aligned}
\sigma_{\mathscr{I}}(t, s)= & \frac{d k_{\mathrm{RD}}(t, s)}{d t}+\frac{d k_{\mathrm{RD}}(s, s)}{d s}-r_{\mathscr{K}}^{\mathrm{I}}(t, s) \\
& -r_{\mathscr{J}}(t, s),
\end{aligned}
$$

where $r_{\mathscr{I}}$ is the Interest Payment Density at time $t$ which is associated with the borrowed amount at time $s$, and where $r_{\mathscr{K}}^{\mathrm{I}}$ is a repayment scheme at time $t$ which is associated with the borrowed amount at time $s$.

In what follows, we will show that the definition of Algebraic Spending Density $\sigma_{\mathscr{g}}$ is consistent with the definition of Algebraic Spending $\sigma$ which is given in relation (6). Indeed, Algebraic Spending Density $\sigma$ can be expressed in terms of $\sigma_{\mathscr{f}}$. By integration over variable $s$ (which describes the borrowed time), from $\sigma_{\mathscr{I}}(t, s)$, Algebraic Spending Density $\sigma$ can be defined as follows:

$$
\sigma(t)=\int_{t_{\mathrm{I}}}^{t} \sigma_{\mathscr{F}}(t, s) d s+\sigma^{I}(t)
$$

where density $\sigma^{I}$ is to be determined, which is Algebraic Spending Density associated with $\mathscr{K}_{\mathrm{RD}}\left(t_{\mathrm{I}}\right)$ the known Current Debt at initial time $t_{\mathrm{I}}$.

$$
\begin{aligned}
\sigma(t)= & -\int_{t_{\mathrm{I}}}^{t} \gamma(t-s) \kappa_{E}(s) d s+\int_{t_{\mathrm{I}}}^{t} d \kappa_{E} \\
& -\int_{t_{\mathrm{I}}}^{t} r_{\mathscr{K}}^{\mathrm{I}}(t, s) d s-\int_{t_{\mathrm{I}}}^{t} r_{\mathscr{I}}(t, s) d s+\sigma^{I}(t) .
\end{aligned}
$$

Replacing first term in relation (86) by $-\kappa_{E} \star \gamma$ and using the fact that the Interest Payment Density $\rho_{\mathscr{F}}$ is expressed in [2] as follows:

$$
r_{\mathcal{F}}(t, s)=\alpha(s) \kappa_{E}(s)-\alpha(s) \int_{s}^{t} \gamma(y-s) \kappa_{E}(s) d y,
$$

we get:

$$
\begin{aligned}
\sigma(t)= & \kappa_{E}(t)-\kappa_{E} \star \gamma(t)-\kappa_{E}\left(t_{\mathrm{I}}\right)-\int_{t_{\mathrm{I}}}^{t} r_{\mathscr{K}}^{\mathrm{I}}(t, s) d s \\
& -\int_{t_{\mathrm{I}}}^{t} \alpha(s) \kappa_{E}(s) d s \\
& +\int_{t_{\mathrm{I}}}^{t} \alpha(s)\left(\int_{s}^{t} \gamma(y-s) \kappa_{E}(s) d y\right) d s \\
& +\sigma^{I}(t) .
\end{aligned}
$$

The integration of Density $r_{\mathscr{K}}^{\mathrm{I}}$ over variable $s$ gives

$$
\rho_{\mathscr{K}}^{\mathrm{I}}(t)=\int_{t_{\mathrm{I}}}^{t} r_{\mathscr{K}}^{\mathrm{I}}(t, s) d s+\rho_{\mathscr{K}}^{\mathrm{I}}\left(t_{\mathrm{I}}\right) .
$$

Expression (88) and consequently definition of Borrowed Time Related Algebraic Spending Density $\sigma_{\mathscr{I}}$ are consistent with the expression of Algebraic Spending Density $\sigma$ given in relation (6). Indeed, if the rate $\alpha(t)$ is fixed with worth $\alpha,(88)$ writes

$$
\begin{aligned}
\sigma(t)= & \kappa_{E}(t)-\kappa_{E} \star \gamma(t)-\kappa_{E}\left(t_{\mathrm{I}}\right)-\int_{t_{\mathrm{I}}}^{t} r_{\mathscr{K}}^{\mathrm{I}}(t, s) d s \\
& -\alpha \int_{t_{\mathrm{I}}}^{t} \kappa_{E}(s) d s \\
& +\alpha \int_{t_{\mathrm{I}}}^{t}\left(\int_{s}^{t} \gamma(y-s) \kappa_{E}(s) d y\right) d s+\sigma^{I}(t) \\
= & \kappa_{E}(t)-\kappa_{E} \star \gamma(t)-\rho_{\mathscr{K}}^{\mathrm{I}}(t) \\
& -\alpha \int_{t_{\mathrm{I}}}^{t}\left(\kappa_{E}(s)-\int_{t_{\mathrm{I}}}^{y} \gamma(y-s) \kappa_{E}(s) d s\right) d y \\
& -\kappa_{E}\left(t_{I}\right)+\rho_{\mathscr{K}}^{\mathrm{I}}\left(t_{\mathrm{I}}\right)+\sigma^{I}(t) \\
= & \kappa_{E}(t)-\kappa_{E} \star \gamma(t)-\alpha \int_{t_{\mathrm{I}}}^{t}\left(\kappa_{E}-\kappa_{E} \star \gamma\right)(s) d s \\
& -\rho_{\mathscr{K}}^{\mathrm{I}}(t)-\kappa_{E}\left(t_{\mathrm{I}}\right)+\rho_{\mathscr{K}}^{\mathrm{I}}\left(t_{\mathrm{I}}\right)+\sigma^{I}(t),
\end{aligned}
$$


where density $\sigma^{I}$ can be expressed as follows:

$$
\sigma^{I}(t)=\kappa_{E}\left(t_{\mathrm{I}}\right)-\rho_{\mathscr{K}}^{\mathrm{I}}\left(t_{\mathrm{I}}\right)-\alpha \int_{t}^{\Theta_{\max }} \rho_{\mathscr{K}}^{\mathrm{I}}(s) d s .
$$

We will justify the expression (91) of density $\sigma^{I}$ as follows. Indeed, replacing time $t$ by initial time $t_{\mathrm{I}}$ in expressions (6) and (91) of Density $\sigma$, we obtain the same expression $\sigma\left(t_{\mathrm{I}}\right)$ defined by

$$
\begin{aligned}
\sigma\left(t_{\mathrm{I}}\right)= & \kappa_{E}\left(t_{\mathrm{I}}\right)-\kappa_{E} \star \gamma\left(t_{\mathrm{I}}\right)-\rho_{\mathscr{K}}^{\mathrm{I}}\left(t_{\mathrm{I}}\right) \\
& -\alpha \int_{t_{\mathrm{I}}}^{\Theta_{\max }} \rho_{\mathscr{K}}^{\mathrm{I}}(s) d s .
\end{aligned}
$$

\section{Inverse Problem of the Model in $\mathbb{S}\left(\mathbb{R}_{+}\right)$}

Denoting $\mathbb{S}\left(\mathbb{R}_{+}\right)$the Schwartz space consists of smooth functions whose derivatives (including the function itself) decay at positive infinity faster than any power. We say, for short, that Schwartz functions are rapidly decreasing. We state the Repayment Pattern Density $\gamma$ as follows:

$$
\gamma \in \mathbb{S}\left(\mathbb{R}_{+}\right) .
$$

We use the Fourier Transform which are operators acting on densities over $\mathbb{R}$. Operators $\mathscr{F}$ stand for the Fourier Transform, and $\mathscr{F}^{-1}$ stands for the Inverse Fourier Transform.

Lemma 3. If function $\kappa_{E}$ is in $\mathbb{S}\left(\mathbb{R}_{+}\right)$and if $\gamma$ satisfies relation (93), then we have the following equality:

$$
\begin{aligned}
(1 & -\mathscr{F}(\gamma)) \mathscr{F}\left(\kappa_{E}\right) \\
& =\mathscr{F}\left(\mathscr{L}\left[\kappa_{E}\right]+\alpha \int_{t_{\mathrm{I}}}^{\bullet} \mathscr{L}\left[\kappa_{E}\right](s) \mathrm{e}^{\alpha(\cdot-s)} d s\right) .
\end{aligned}
$$

Proof. Integrating by parts states that

$$
\begin{gathered}
\int_{t_{\mathrm{I}}}^{t}\left(\int_{t_{\mathrm{I}}}^{s}\left(\kappa_{E}-\kappa_{E} \star \gamma\right)(y) d y\right) \times \alpha(t) \mathrm{e}^{\alpha(t-s)} d s \\
=\int_{t_{\mathrm{I}}}^{t}\left(\kappa_{E}-\kappa_{E} \star \gamma\right)(s) \times \mathrm{e}^{\alpha(t-s)} d s \\
\quad-\int_{t_{\mathrm{I}}}^{t}\left(\kappa_{E}-\kappa_{E} \star \gamma\right)(s) d s .
\end{gathered}
$$

From this, we get the following equality:

$$
\begin{aligned}
& \int_{t_{\mathrm{I}}}^{t}\left(\kappa_{E}-\kappa_{E} \star \gamma\right)(s) d s=\int_{t_{\mathrm{I}}}^{t}\left(\left(\kappa_{E}-\kappa_{E} \star \gamma\right)(s)\right. \\
& \left.-\alpha(t) \int_{t_{\mathrm{I}}}^{s}\left(\kappa_{E}-\kappa_{E} \star \gamma\right)(y) d y\right) \times \mathrm{e}^{\alpha(t)(t-s)} d s .
\end{aligned}
$$

Using definition (18) of operator $\mathscr{L}$, equality in (96) is multiplied by density $\alpha(t)$ to give

$$
\begin{aligned}
& \alpha(t) \int_{t_{\mathrm{I}}}^{t}\left(\kappa_{E}-\kappa_{E} \star \gamma\right)(s) d s \\
& =\alpha(t) \int_{t_{\mathrm{I}}}^{t} \mathscr{L}\left[\kappa_{E}\right](s) \mathrm{e}^{\alpha(t-s)} d s .
\end{aligned}
$$

Replacing $\alpha(t) \int_{t_{\mathrm{T}}}^{t}\left(\kappa_{E}-\kappa_{E} \star \gamma\right)(s) d s$ in relation (97) by $\left(\kappa_{E}-\right.$ $\left.\kappa_{E} \star \gamma\right)(t)-\mathscr{L}\left[\kappa_{E}\right](t)$ which is possible because of (18), we obtain the following equality:

$$
\begin{aligned}
\kappa_{E}(t)-\kappa_{E} \star \gamma(t)= & \mathscr{L}\left[\kappa_{E}\right](t) \\
& +\alpha(t) \int_{t_{\mathrm{I}}}^{t} \mathscr{L}\left[\kappa_{E}\right](s) \mathrm{e}^{\alpha(\cdot-s)} d s .
\end{aligned}
$$

Applying Fourier Transform to each term of equality (98), we obtain equality (94), proving the lemma.

Lemma 4. Assuming (93), that implies that

$$
\int_{0}^{+\infty} y \gamma(y) d y \neq 0
$$

is achieved and if function $\mathscr{L}\left[\kappa_{E}\right]$ given by relation (7) satisfies

$$
\begin{aligned}
& \int_{0}^{+\infty}\left(\mathscr{L}\left[\kappa_{E}\right](y)+\alpha \int_{0}^{y} \mathscr{L}\left[\kappa_{E}\right](s) \mathrm{e}^{\alpha(y-s)} d s\right) d y \\
& \quad=0
\end{aligned}
$$

and for a negative rate $\alpha$

$$
t \longmapsto \int_{0}^{t} \mathscr{L}\left[\kappa_{E}\right](s) d s \in \mathbb{S}\left(\mathbb{R}_{+}\right)
$$

then, $\mathscr{F}\left(\kappa_{E}\right) \in \mathbb{L}^{\infty}(\mathbb{R})$ and is such that

$$
\begin{aligned}
& \lim _{\xi \rightarrow 0} \mathscr{F}\left(\kappa_{E}\right)(\xi)=-\frac{2}{\Theta_{\gamma}^{2}} \int_{0}^{+\infty} y \times\left(\mathscr{L}\left[\kappa_{E}\right](y)\right. \\
& \left.+\alpha \int_{0}^{y} \mathscr{L}\left[\kappa_{E}\right](s) \mathrm{e}^{\alpha(y-s)} d s\right) d y .
\end{aligned}
$$

If $\mathscr{L}\left[\kappa_{E}\right]$ does not satisfy the equality in relation (100), then, $\mathscr{F}\left(\kappa_{E}\right)$ has an infinite limit in 0 .

Proof. As $\mathscr{L}\left[\kappa_{E}\right] \in \mathbb{S}\left(\mathbb{R}_{+}\right)$and the fact that $\mathbb{S}\left(\mathbb{R}_{+}\right) \subset \mathbb{L}^{1}\left(\mathbb{R}_{+}\right)$, we get

$$
\mathscr{L}\left[\kappa_{E}\right]+\alpha \int_{0}^{\bullet} \mathscr{L}\left[\kappa_{E}\right](s) \mathrm{e}^{\alpha(\cdot-s)} d s \in \mathbb{L}^{1}\left(\mathbb{R}_{+}\right) .
$$

Indeed, under assumption (101) the product of a bounded function $t \mapsto \mathrm{e}^{\alpha(t-s)}$ due to the negative rate $\alpha$ by a function in $\mathbb{L}^{1}\left(\mathbb{R}_{+}\right)$is a function in $\mathbb{L}^{1}\left(\mathbb{R}_{+}\right)$. Then, using an order 1 
Taylor expansion of $e^{-i y \xi}$, we obtain the following expansion of function $\mathscr{F}\left(\mathscr{L}\left[\kappa_{E}\right]+\alpha \int_{0}^{\bullet} \mathscr{L}\left[\kappa_{E}\right](s) \mathrm{e}^{\alpha(\bullet-s)} d s\right)$ :

$$
\begin{aligned}
\mathscr{F} & \left(\mathscr{L}\left[\kappa_{E}\right]+\alpha \int_{0}^{\bullet} \mathscr{L}\left[\kappa_{E}\right](s) \mathrm{e}^{\alpha(\bullet-s)} d s\right)(\xi) \\
& =\int_{0}^{+\infty}\left(\mathscr{L}\left[\kappa_{E}\right](y)\right. \\
& \left.+\alpha \int_{t_{\mathrm{I}}}^{y} \mathscr{L}\left[\kappa_{E}\right](s) \mathrm{e}^{\alpha(y-s)} d s\right) d y-i \xi \int_{0}^{+\infty} y \\
& \times\left(\mathscr{L}\left[\kappa_{E}\right](y)\right. \\
& \left.+\alpha \int_{0}^{y} \mathscr{L}\left[\kappa_{E}\right](s) \mathrm{e}^{\alpha(y-s)} d s\right) d y+O\left(\xi^{2}\right) .
\end{aligned}
$$

Since operator $\mathscr{L}$ satisfies equality in relation (100), relation (104) gives

$$
\begin{aligned}
& \mathscr{F}\left(\mathscr{L}\left[\kappa_{E}\right]+\alpha \int_{t_{\mathrm{I}}}^{\bullet} \mathscr{L}\left[\kappa_{E}\right](s) \mathrm{e}^{\alpha(\bullet-s)} d s\right)(\xi) \\
& \quad=-i \xi \int_{0}^{+\infty} y \\
& \quad \times\left(\mathscr{L}\left[\kappa_{E}\right](y)+\alpha \int_{t_{\mathrm{I}}}^{y} \mathscr{L}\left[\kappa_{E}\right](s) \mathrm{e}^{\alpha(y-s)} d s\right) d y \\
& \quad+O\left(\xi^{2}\right) .
\end{aligned}
$$

According to (93), function $1-\mathscr{F}(\gamma)$ is Taylor expanded in 0 until the order 1 to obtain

$$
1-\mathscr{F}(\gamma)(\xi)=i \xi \int_{0}^{+\infty} y \gamma(y) d y+O\left(\xi^{2}\right) .
$$

According to relations (94), (105), and (106), we get equality (102). Moreover, according to relation $(94), \mathscr{F}\left(\kappa_{E}\right)$ is a finite quantity outside of 0 . Hence, it is concluded that $\mathscr{F}\left(\kappa_{E}\right)$ is in $\mathbb{L}^{\infty}(\mathbb{R})$. On the other hand, if equality in relation $(100)$ is not satisfied, then, according to relations (94), (104), and (106), $\mathscr{F}\left(\kappa_{E}\right)$ has an infinite limit at 0 . From this, the proof of the lemma is achieved.

Theorem 5. If Repayment Pattern Density $\gamma$ satisfies relation (93) such that

$$
|\mathscr{F}(\gamma)|<1
$$

and if Initial Debt Repayment Density $\rho_{\mathscr{K}}^{\mathrm{I}}$ is in $\mathbb{S}\left(\mathbb{R}_{+}\right)$, then for any Algebraic Spending Density $\sigma$ in $\mathbb{S}\left(\mathbb{R}_{+}\right)$satisfying the following equality:

$$
\begin{aligned}
& \int_{0}^{+\infty}\left(\sigma(y)-\mathscr{D}\left[\rho_{\mathscr{K}}^{\mathrm{I}}\right](y)\right. \\
& \left.\quad+\alpha \int_{0}^{y}\left(\sigma(s)-\mathscr{D}\left[\rho_{\mathscr{K}}^{\mathrm{I}}\right](s)\right) \mathrm{e}^{\alpha(y-s)} d s\right) d y \\
& \quad=0,
\end{aligned}
$$

there exists an unique Loan Density $\kappa_{E}$ in $\mathbb{S}\left(\mathbb{R}_{+}\right)$which is given in terms of $\sigma$ by

$$
\kappa_{E}=\mathscr{F}^{-1}\left(\frac{\mathscr{F}\left(\sigma-\mathscr{D}\left[\rho_{\mathscr{K}}^{\mathrm{I}}\right]+\alpha \int_{0}^{\bullet}\left(\sigma(s)-\mathscr{D}\left[\rho_{\mathscr{K}}^{\mathrm{I}}\right](s)\right) \mathrm{e}^{\alpha(\bullet-s)} d s\right)}{1-\mathscr{F}(\gamma)}\right) .
$$

Proof. According to relation (107), we get

$$
\frac{1}{1-\mathscr{F}(\gamma)}=\sum_{k \geq 0} \mathscr{F}^{k}(\gamma)
$$

In the first place, we will show that the left term given by $(110)$ is in $\mathbb{S}\left(\mathbb{R}_{+}\right)$. Indeed, since $\mathbb{S}\left(\mathbb{R}_{+}\right)$is stable by Fourier Transform, assumption (93) states that $\mathscr{F}(\gamma)$ is in $\mathbb{S}\left(\mathbb{R}_{+}\right)$. From this, quantity $1 /(1-\mathscr{F}(\gamma))$ is in $\mathbb{S}\left(\mathbb{R}_{+}\right)$due to its stability under power and infinite sum. Secondly, we will show that density $\sigma-\mathscr{D}\left[\rho_{\mathscr{K}}^{\mathrm{I}}\right]+\alpha F$ is in $\mathbb{S}\left(\mathbb{R}_{+}\right)$, where density $F$ is defined by

$$
F(t)=\int_{0}^{t}\left(\sigma(s)-\mathscr{D}\left[\rho_{\mathscr{K}}^{\mathrm{I}}\right](s)\right) \mathrm{e}^{\alpha(t-s)} d s
$$

Because densities $\sigma$ and $\mathscr{D}\left[\rho_{\mathscr{K}}^{\mathrm{I}}\right]$ are in $\mathbb{S}\left(\mathbb{R}_{+}\right)$, density $\sigma-$ $\mathscr{D}\left[\rho_{\mathscr{K}}^{\mathrm{I}}\right]$ is rapidly decreasing. Thus, for an integer $N^{\prime}$ there exists a positive constant $C_{N^{\prime}}$ such that

$$
\sigma(s)-\mathscr{D}\left[\rho_{\mathscr{K}}^{\mathrm{I}}\right](s) \leq \frac{C_{N^{\prime}}}{(1+s)^{N^{\prime}}}, \quad \forall s \in \mathbb{R}_{+}
$$

Since the derivative of density $F$ is equal to $\sigma-\mathscr{D}\left[\rho_{\mathscr{K}}^{\mathrm{I}}\right]$, inequality (112) gives

$$
F^{\prime}(s) \leq \frac{C_{N^{\prime}}}{(1+s)^{N^{\prime}}}, \quad \forall s \in \mathbb{R}_{+}
$$

Integrate inequality $(113)$ over $[0, t]$ to get

$$
F(t) \leq \frac{C_{N^{\prime}}}{N^{\prime}-1}\left(1-\frac{1}{(1+t)^{N^{\prime}-1}}\right), \quad \forall t \in \mathbb{R}_{+}
$$


Consequently, for an integer $N$ there exists a positive constant $C_{N}$ such that

$$
F(t) \leq \frac{C_{N}}{(1+t)^{N}}, \quad \forall t \in \mathbb{R}_{+} .
$$

Inequality (115) states that $F$ is rapidly decreasing. Furthermore, since the $(l+1)$-nd derivative of density $F$ is equal to the $l$-nd derivative of density $\sigma-\mathscr{D}\left[\rho_{\mathscr{K}}^{\mathrm{I}}\right]$,

$$
F^{(l+1)}(t)=\left(\sigma-\mathscr{D}\left[\rho_{\mathscr{K}}^{\mathrm{I}}\right]\right)^{(l)}(t), \quad \forall l \in \mathbb{N}, \forall t \in \mathbb{R}_{+},
$$

and the fact that space $\mathbb{S}\left(\mathbb{R}_{+}\right)$is stable under the operation of derivation, for any integer $l$ function $F^{(l+1)}$ is rapidly decreasing. Consequently, $F$ is in $\mathbb{S}\left(\mathbb{R}_{+}\right)$, achieving the second point of proof. We conclude that

$$
\begin{aligned}
& \frac{\mathscr{F}\left(\sigma-\mathscr{D}\left[\rho_{\mathscr{K}}^{\mathrm{I}}\right]+\alpha \int_{0}^{\bullet}\left(\sigma(s)-\mathscr{D}\left[\rho_{\mathscr{K}}^{\mathrm{I}}\right](s)\right) \mathrm{e}^{\alpha(\bullet-s)} d s\right)}{1-\mathscr{F}(\gamma)} \\
& \quad \in \mathbb{S}\left(\mathbb{R}_{+}\right)
\end{aligned}
$$

because it is the product of two functions in $\mathbb{S}\left(\mathbb{R}_{+}\right)$.

\section{Conflicts of Interest}

The author declares that there are no conflicts of interests.

\section{References}

[1] J. Hadamard, Lectures on Cauchy's Problem in Linear Partial Differential Equations, New Haven Yale University Press, 1923.

[2] E. Frenod and T. Chakkour, "A continuous-in-time financial model," Mathematical Finance Letters, vol. 2016, 2016.

[3] T. Chakkour, "Implementing some mathematical operators for a continuous-in-time financial model," Engineering Mathematics Letters, vol. 2017, article 2, 2017.

[4] T. Chakkour and E. Frenod, "Inverse problem and concentration method of a continuous-in-time financial model," International Journal of Financial Engineering, vol. 3, no. 2, Article ID 1650016, 20 pages, 2016.

[5] F. Bauer and M. A. Lukas, "Comparingparameter choice methods for regularization of ill-posed problems," Mathematics and Computers in Simulation, vol. 81, no. 9, pp. 1795-1841, 2011.

[6] P. C. Hansen, "The discrete Picard condition for discrete illposed problems," BIT. Numerical Mathematics, vol. 30, no. 4, pp. 658-672, 1990.

[7] R. Hempel and J. Voigt, "The spectrum of a schrödinger operator in $l p(\mathbb{R} v)$ is p-independent," Communications in Mathematical Physics, vol. 104, no. 2, pp. 243-250, 1986.

[8] K. Chadan, D. Colton, W. Rundell, and L. Päivärinta, An Introduction to Inverse Scattering and Inverse Spectral Problems, vol. 2 of SIAM Monographs on Mathematical Modeling and Computation, SIAM, Philadelphia, Pa, USA, 1997.

[9] M. I. Gil', "Invertibility and spectrum localization of non-selfadjoint operators," Advances in Applied Mathematics, vol. 28, no. 1, pp. 40-58, 2002.

[10] M. I. Gil', Operator Functions and Localization of Spectra, vol. 1830 of Lecture Notes in Mathematics, Springer, Berlin, Germany, 2003.
[11] A. Gupta and B. S. Komal, "Volterra composition operators," International Journal of Contemporary Mathematical Sciences, vol. 6, no. 5-8, pp. 345-351, 2011. 


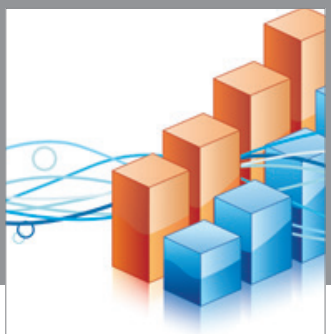

Advances in

Operations Research

vatem alat4

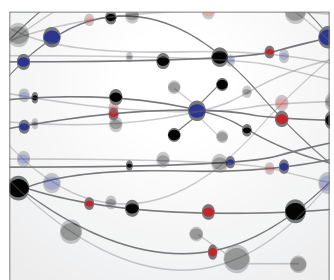

\section{The Scientific} World Journal
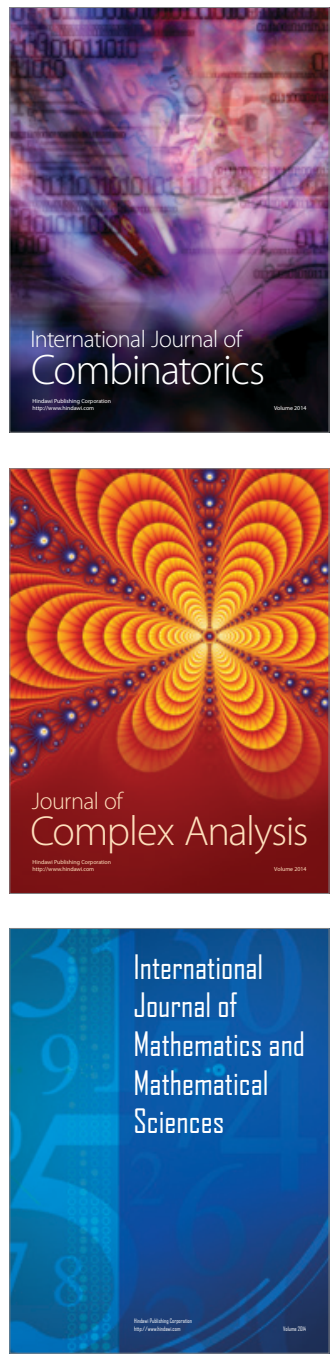
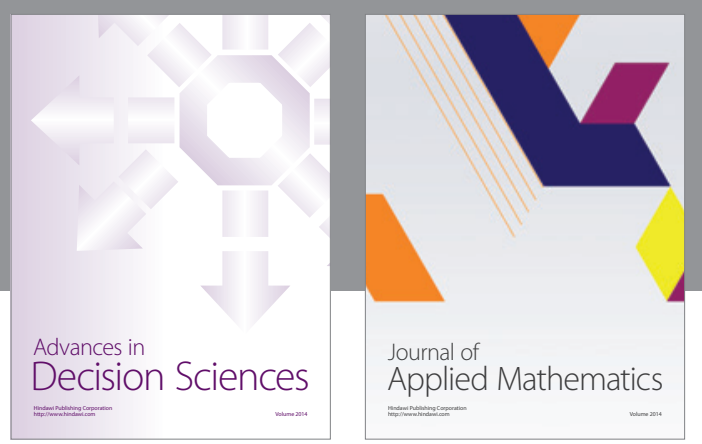

Algebra

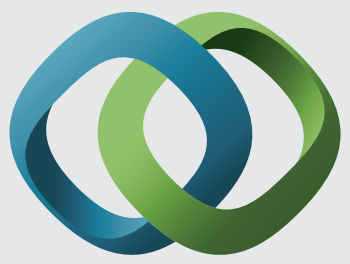

\section{Hindawi}

Submit your manuscripts at

https://www.hindawi.com
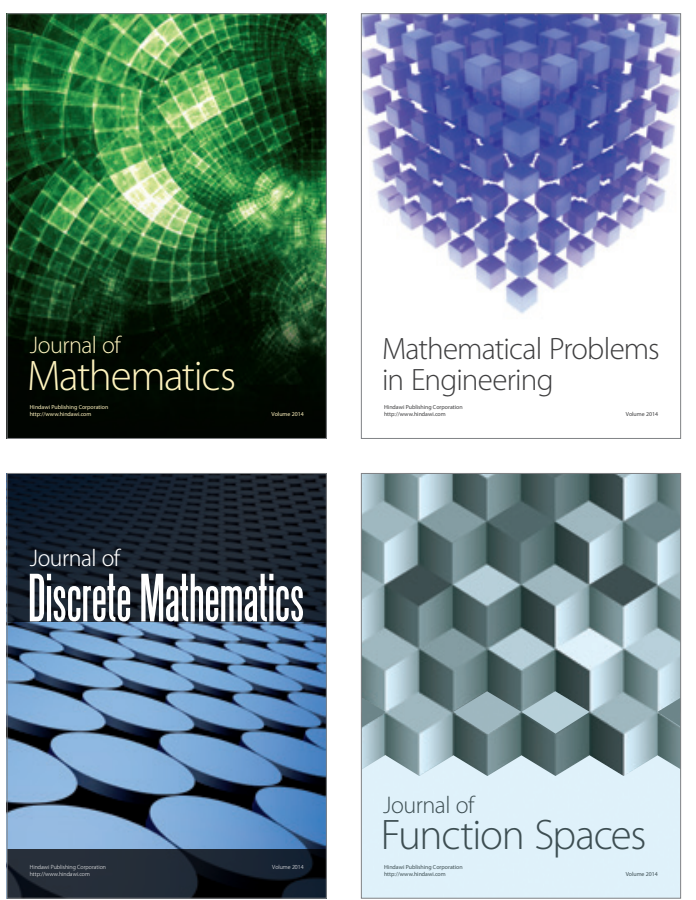

Mathematical Problems in Engineering
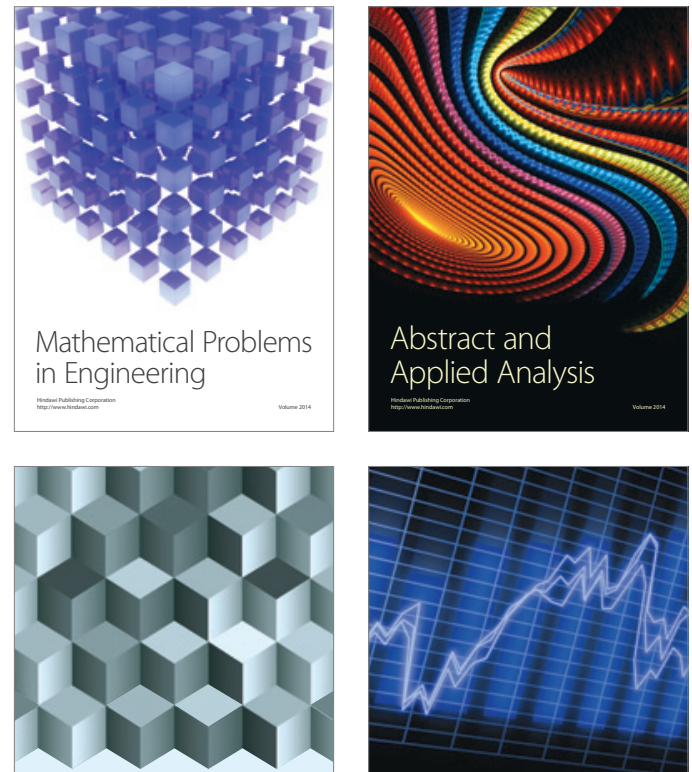

Journal of

Function Spaces

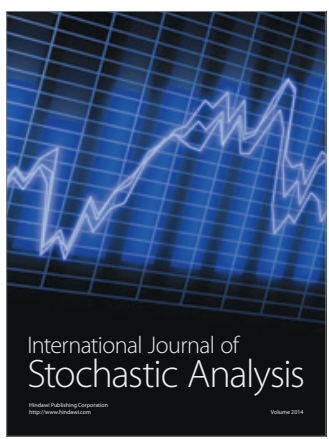

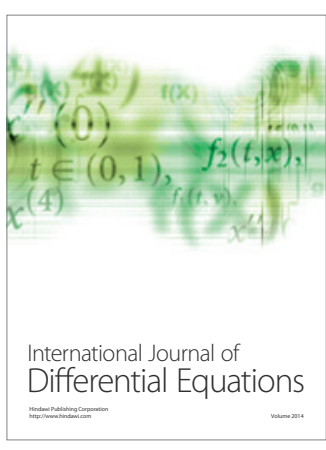
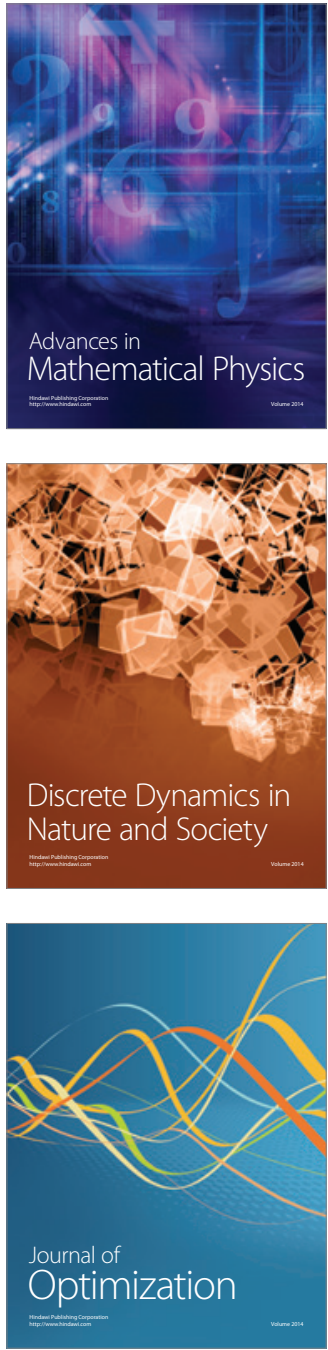